MATEC Web of Conferences 48,01002 (2016)

DOI: $10.1051 /$ matecconf $/ 20164801002$

(C) Owned by the authors, published by EDP Sciences, 2016

\title{
Hybrid gravitational orientation system of small spacecraft
}

\author{
Victor Dmitriev ${ }^{1}$, Ruslan Frolov ${ }^{1 . a}$ and Anastasia Bekasova ${ }^{1}$ \\ ${ }^{1}$ National Research Tomsk Polytechnic University, 634050 Tomsk, Russia
}

\begin{abstract}
In this article is considered the problem of small satellite's angular position control. This problem is solved by some improvement. The authors offer to use active control element, in the form of gyrodyne, which to fix on the end of gravitational rod. This improvement transforms the gravitational system of orientation into a control system along three axes of orientation: yaw, roll and pitch.
\end{abstract}

\section{Introduction}

The principle of gravitational stabilization of an object caused by the gradient of the gravitational field of the Earth has been known since the Lagrange's work about libration of the Moon published in 1780. He determined the conditions of stable oscillations of the body in vertical orientation of its longitudinal axis.

Thus, stabilization of the artificial earth satellite relative to the local vertical line can be realized using the properties of the Earth's gravitational field gradient with simultaneous damping of spacecraft librations without active technical control systems employed.

Under certain conditions (similar to the constant direction of the Moon relative to the Earth), a specified ratio of the moments of inertia of the spacecraft body will make the axis of smallest moment of inertia be constantly directed towards the center of the Earth.

The main advantage of the above orientation is that the aerospace directional antenna used for amplification of the spacecraft radiofrequency signal will by constantly directed to the center of the Earth.

Due to problems directly related to the development of space technology and the rate of technological evolution, the fundamental works on gravitational systems were published after 2000 [1-4].

\section{Problem of modern gravitational orientation systems}

The gravitational systems of orientation consist of a gravitational rod with load attached at its end. The shape of this small spacecraft can be considered as a dumbbell shape (the rod with a spacecraft and load fixed at its ends). During orbital flight, this spacecraft becomes the axis of the minimum moment of inertia perpendicular to the center of the Earth (figure 1). However, this method provides stabilization of the spacecraft only along 2 axes - pitch and roll. Along the yaw axis, the spacecraft remains free to spontaneously rotate about this axis at any angles. In some cases, this limits the

\footnotetext{
${ }^{a}$ Corresponding author : phrolov1994@mail.ru
} 
performance of the spacecraft, for example, orientation of solar panels along the normal to the direction of sunlight.

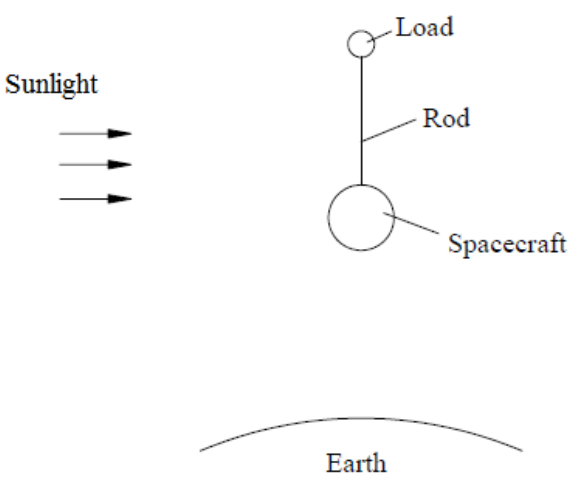

Figure 1. Principle of gravitational stabilization.

The main fundamental disadvantage of all gravitational systems of orientation is that passive gravitational systems of orientation are stabilizing systems of the angular position of the spacecraft, but not controlling ones.

\section{Technical decision of the problem}

This problem can be solved with different types of combined orientation systems [5]. This paper discusses a hybrid gravitational system of orientation of small spacecraft, where a two-axis gyroscope (gyrodyne) implements the function of the load. Its kinematic momentum is directed along the normal to the orbit plane, and the axis of the suspension is placed in the orbit plane, as shown in figure 2 . This improvement transforms the gravitational system of orientation into a control system along three axes of orientation: yaw, roll and pitch.

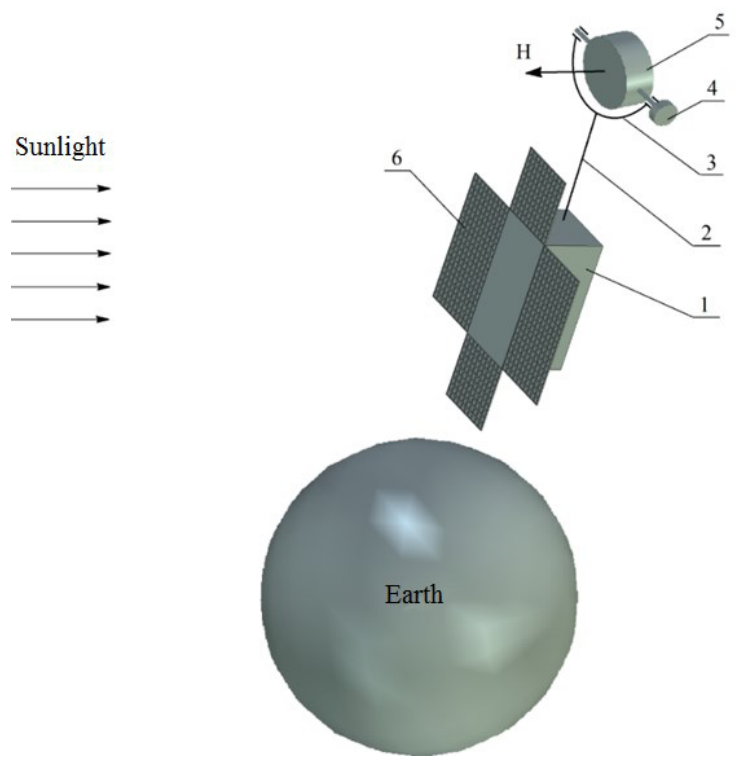

Figure 2. Kinematic scheme of the mechanical system "spacecraft - rod-gyrodyne". 
Figure 2 shows the gravitational system of orientation, which consists of spacecraft (1); rod (2); suspension (3); torque sensor (4); gyromotor (engine-flywheel) (5); solar power panel (6).

Rod 2, which turns from the transport position to the working one, is fastened to spacecraft housing 1 (figure 2). The gyrodyne is fastened at the end of the rod and consists of gyromotor 5, frame 3 and torque sensor 4 . In this case, the spacecraft will be stabilized along the roll and pitch positions by means of the gravitational moment from rod 2. The angle yaw relative to the axis of the rod provides the control of the angular position of the spacecraft via the gyroscopic moment when power is supplied to torque sensor 4 .

The gyrodyne (figure 3) consists of housing 2 fastened to rod 1, wherein gyromotor 3 is attached by semi-axes 4 . In the general case, the vector of the gyromotor kinematic momentum is directed perpendicularly to the orbit plane. Semi-axes 4 can rotate in housing 2 through bearing supports 6 . Torque sensor 5 is attached to the semi-axes of the suspension element.

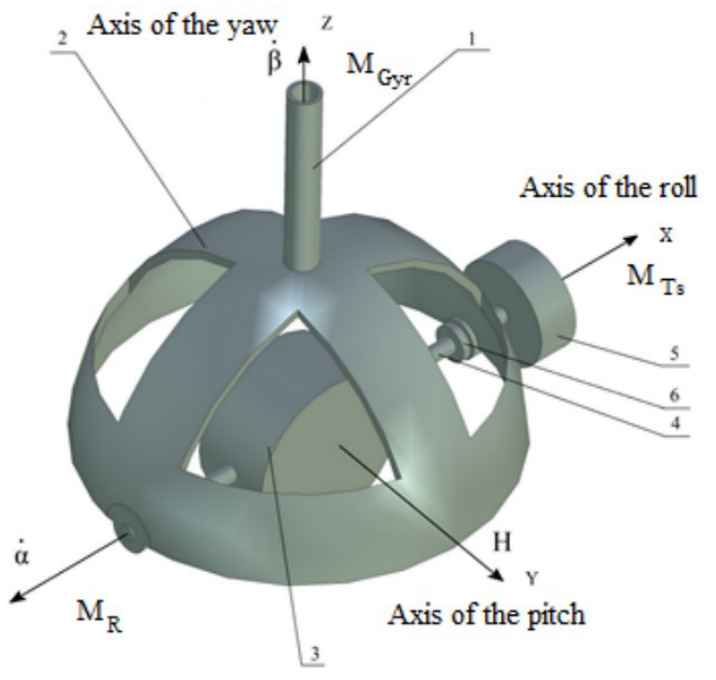

Figure 3. General arrangement of the gyrodyne.

When power is supplied to the sensor stator winding in accordance with the theory of gyroscopes under the law of the precession relative to the gravitational rod axis, the gyroscopic moment $\mathrm{M}_{\mathrm{Gyr}}$ appears and this causes angular motion of the mechanical system "gyromotor- spacecraft - rod" relative to the axis of the rod with angular rate $\dot{\beta}$.

The presence of the angular rate $\dot{\beta}$ relative to the axis of the rod causes the moment $M_{R}$, which is equal to the moment $\mathrm{M}_{\mathrm{Ts}}$. The moment $\mathrm{M}_{\mathrm{Ts}}$ is produced by torque sensors, and it is oppositely directed, i.e. $\mathrm{M}_{\mathrm{Ts}}=-\mathrm{M}_{\mathrm{R}}$.

Cutoff of power supply to the torque sensor via the control unit stops mechanical action on the spacecraft $\dot{\beta}$. In this case, stabilization of the spacecraft angular position is performed by the gravitational moment produced by the rod and kinematic momentum $\mathrm{H}$ of the gyroscope.

\subsection{Principles of control}

Attitude control can be fulfilled different ways [6].Three-axis control of the considered spacecraft is performed as follows.

Stabilization relative to the roll and pitch axes is achieved by the gravitational principle. Angle yaw control is performed under the law of precession as noted above. In addition, the feature of the gyrodyne is the variable speed of the gyromotor, i.e. the gyromotor is a cosmic engine-flywheel $[7,8]$. 
Jaw control and realization of the required direction of the small spacecraft with adequate accuracy is performed as follows.

Figure 4 shows the area on the surface of the Earth divided into four quadrants. The objects to be scanned are dots 1 and 2 . The scanning device is directed to dot " 0 " with the account of the precision of the gravitational system of orientation.

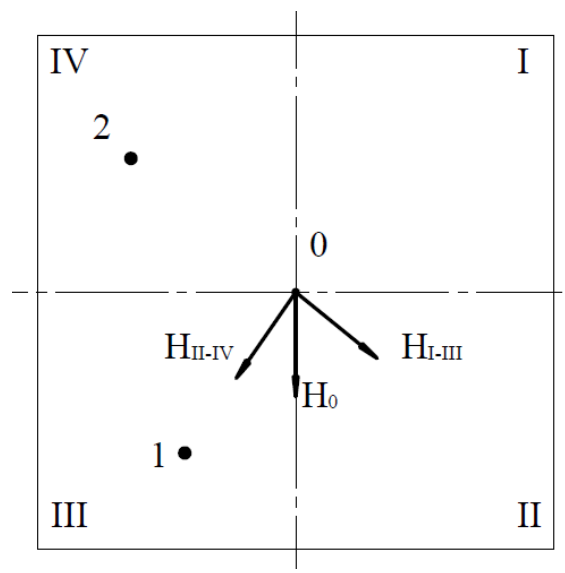

Figure 4.Graphical representation of the gravitational rod axis displacement on the Earth`s surface.

For targeting at dot 1 , the torque sensor is turned on. It rotates the small spacecraft about the gravitational rod axis, and the momentum $\mathrm{H}$ of the gyrodyne moves from position $\mathrm{H}_{0}$ to position $\mathrm{H}_{\mathrm{I}-\mathrm{III}}$. As a result, dot 1 holds the position in the line, which is perpendicular to the momentum. After that, the regulated-velocity engine-flywheel is turned on (gyromotor 3). When electromechanical torque changes its magnitude, the equipment of the small spacecraft will be targeted at position 1, or any other position inside I or III quadrants.

To target the small spacecraft at quadrants II or IV, the momentum $\mathrm{H}$ is displaced from position $\mathrm{H}_{0}$ to position $\mathrm{H}_{\mathrm{II}-\mathrm{IV}}$. After that, the flywheel targets the device sat dot 2 or any other dot inside quadrants I or III.

When the small spacecraft, which is cylindrically symmetrical and has different moments of inertia, moves inside the central Earth's gravitational field, itis affected by torques, which stabilize it in the Earth's coordinate system (figure 5). These torques can be approximately determined by the following expressions:

in the yaw plane

$$
M_{z}=\frac{1}{2} \omega_{0}^{2}\left(J_{x}-J_{y}\right) \sin 2 \psi
$$

in the pitch plane

$$
M_{x}=\frac{3}{2} \omega_{0}^{2}\left(J_{z}-J_{y}\right) \sin 2 \varphi
$$

in the roll plane

$$
M_{y}=\frac{3}{2} \omega_{0}^{2}\left(J_{z}-J_{x}\right) \sin 2 \vartheta
$$

$\mathrm{J}_{\mathrm{x}}, \mathrm{J}_{\mathrm{y}}, \mathrm{J}_{\mathrm{z}}$ are the main central moments of inertia of the small spacecraft;

$\omega_{0}$ is the orbital rate of the small spacecraft;

$\vartheta, \varphi, \psi$ are the angles of pitch, roll and yaw.

These expressions show that restoring torque depends on the difference between the moments of inertia relative to the axes (direct relation), and this torque increases as a sinus of the double angle between the actual and null position of the gravitational rod axis. 


\subsection{Dynamics analysis of control}

Full differential equations of motion of the mechanical system "small spacecraft - rod - gyrodyne" obtained using the second method of Lagrange are complex and interrelated.

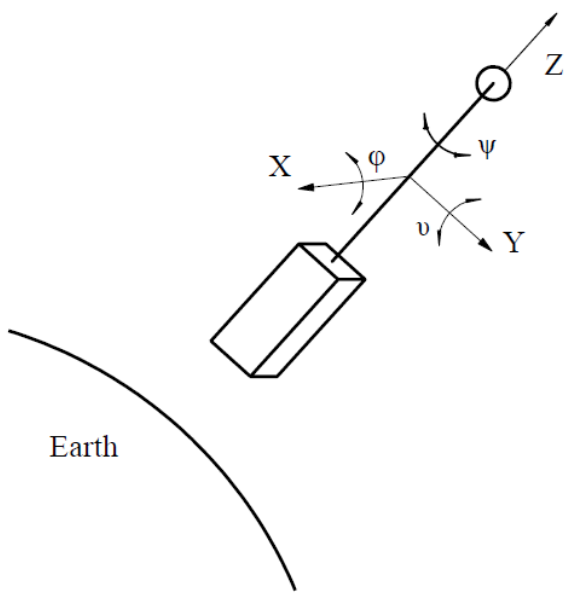

Figure 5. Schematic diagram of the gravitational system of orientation.

After separation from the mother missile, the spacecraft passes through a calm stage. It is affected by the gravity and gyroscopic moments and makes oscillations of small magnitude and rate. Therefore the roll, yaw and pitch positions of the small spacecraft, as well as the angular velocity with respect to these axes can be considered negligible, and the simplified equations take the form $[4,9]$ :

$$
\begin{gathered}
J_{x} \cdot \ddot{\varphi}+\left(J_{y} \cdot \omega_{0}-J_{F} \cdot \omega_{F}\right) \cdot \dot{\psi}+\left[3 \cdot\left(J_{z}-J_{y}\right) \cdot \omega_{0}{ }^{2}+J_{F} \cdot \omega_{F} \cdot \omega_{0}\right] \cdot \varphi+J_{F} \cdot \dot{\omega}_{F} \cdot \psi=0 \\
J_{y} \cdot \ddot{\psi}+\left(J_{F} \cdot \omega_{F}-J_{y} \cdot \omega_{0}\right) \cdot \dot{\varphi}+\left[3 \cdot\left(J_{z}-J_{x}\right) \cdot \omega_{0}{ }^{2}+J_{F} \cdot \omega_{F} \cdot \omega_{0}\right] \cdot \psi+J_{F} \cdot \dot{\omega}_{F} \cdot \varphi=0 \\
J_{z} \cdot \ddot{\vartheta}+\omega_{0}{ }^{2} \cdot\left(J_{x}-J_{y}\right) \cdot \vartheta+J_{F} \cdot \dot{\omega}_{F} \cdot \dot{\alpha}=0 \\
J_{e} \cdot \ddot{\alpha}=M_{G}+J_{F} \cdot \omega_{F} \cdot \dot{\vartheta}+J_{F} \cdot \omega_{F} \cdot \dot{\psi}
\end{gathered}
$$

$\mathrm{J}_{\mathrm{x}}, \mathrm{J}_{\mathrm{y}}, \mathrm{J}_{\mathrm{z}}$ are the main central moments of inertia of the small spacecraft;

$\mathrm{J}_{\mathrm{F}}$ is the momentum of inertia of the flywheel;

$\omega_{F}$ is the angular rate of the flywheel;

$\omega_{0}$ is the angular orbital rate of rotation of the small spacecraft;

$\dot{\alpha}, \ddot{\alpha}$ are the angular acceleration and rate of the engine-flywheel in the uniaxial suspension element;

$\mathrm{J}_{\mathrm{e}}$ is the equatorial moment of inertia of the flywheel.

To simplify the system of differential equations (2-7) and to gain better understanding of the dynamics of the mechanical system, some members are neglected. These are, for example, members that depend of damping properties of the rod material, construction material, active damping devices, which dissipate the kinetic energy of the rotational motion, and bending oscillations of the gravitational rod near null position.

Equations (2-7) show that the yaw motion of the small spacecraft in the orbital plane is not related to the roll and pitch motions. Application of the gyrodyne with the regulated-velocity engine-flywheel allows control along the yaw, roll and pitch axes and increases restoring torque. 


\section{Conclusion}

The paper describes a hybrid gravitational orientation system of small spacecraft which uses a biaxial gyroscope with the regulated-velocity flywheel firmly fixed at the end of the gravitational rod as load. It significantly expands the functionality of this orientation system.

This system is hybrid as it combines the main advantage of the passive system (minimization of energy consumption during pauses in operation of the payload) and remains constantly directed towards the Earth's surface.

This gravitational orientation system is provided with software to perform targeting during operation with adequate accuracy. The accuracy of the gravitational system of orientation is about $(5-10)^{\circ}$, while the hybrid system in the active mode can realize the accuracy within arcminutes.

Since the equipment does not work all the period of the aircraft operation, energy is saved, and this is crucial for small spacecrafts.

Moreover, this orientation system has an operational advantage: replacement of the passive load to an active control element of equal mass.

\section{References}

1. Y. Wang, S. Xu, J. Aerospace Eng. 27, 141 (2014)

2. S. A. Rawashdeh, University of Kentucky Master's Theses, 624 (2010)

3. A. M. Si Mohammed, M. Benyettou, Y. Bentoutou, A. Boudjemai, Y. Hashida, M. N. Sweeting, Acta Astron. 64, 802 (2009)

4. H. J. Fletcher, L. Rongved, E. Y. Yu, Bell Syst. Tech. J. 42,2245 (2013)

5. C. Circi, J. Aerospace Eng. 221, 87 (2007)

6. Godard, K. D. Kumar, Guidance, Navigation, and Control Conference, 8197 (2010)

7. D. J. Richie,V. J. Lappas, B. Wie, ASCE, 7501 (2008)

8. H. Yoon, P. Tsiotras, J. Guid. Control Dyn. 25, 1084 (2002)

9. T. Pulecchi, F. Casella, M. Lovera, Simul. Model. Pract. Th. 18,77 (2010) 